\title{
Mean-value theorems for multiplicative functions on Beurling's generalized integers (I)
}

\section{Wen-Bin Zhang}

Published online: 8 October 2008

(C) Springer-Verlag 2008

\section{Erratum to: Math. Z. (2005) 251:359-391 \\ DOI 10.1007/s00209-005-0807-8}

It has been discovered that, in my paper [2], quoted in the title of this correction, the condition (1.4) on the Chebyshev function $\psi(x)$ of Theorem 2, the same condition quoted in the abstract, and the condition on the same function $\psi(x)$ in the remark following Corollary 1 are incorrect in their current formulation. The mistake is due to an incorrect citation from [1].

To derive the correction of the conditions, we begin with Beurling's theorems. Suppose that the counting function $N(x)$ of Beurling's generalized integers $\mathcal{N}$ satisfies

$$
N(x)=x \sum_{r=1}^{m} A_{r}(\log x)^{\rho_{r}-1}+O\left(x \log ^{-\gamma} x\right)
$$

with a constant $\gamma>0$, real constants $A_{1}, \ldots, A_{m}$ such that $A_{m}=A>0$, and contants $\rho_{1}<\rho_{2}<\cdots<\rho_{m}$ such that $\rho_{m}=\rho \geq 1$. Then, for $\sigma>1$,

$$
\zeta(s):=\int_{1-}^{\infty} x^{-s} d N(x)=\sum_{j=0}^{\infty} \frac{1}{n_{j}^{s}}
$$

is the zeta function associated with $\mathcal{N}$. The Euler product formula implies that $\zeta(s)$ has no zeros for $\sigma>1$. Beurling [1] showed that if $\gamma>1+\rho / 2$ then the degree $\alpha(t)$ of a zero $1+i t$ of $\zeta(s)$ on the vertical line $\sigma=1$ is a positive integer and that the total number of such

The online version of the original article can be found under doi:10.1007/s00209-005-0807-8.

W.-B. Zhang $(\varangle)$

Department of Mathematics, University of Illinois, 1409 West Green Street, Urbana, Illinois 61801, USA

e-mail: w-zhang1@math.uiuc.edu

W.-B. Zhang

Department of Mathematics, University of The West Indies, Mona, Kingston 7, Jamaica 
zeros does not exceed $\rho$ in the sense that

$$
\sum_{t_{j}>0} \alpha\left(t_{j}\right) \leq \rho / 2
$$

Hence there exist only a finite number of such zeros $1 \pm i t_{j}, j=1, \ldots, \ell$. Beurling showed $[1$, p. 263] also that the counting function $\pi(x)$ of the generalized primes satisfies

$$
\Pi(x) \sim v \cdot p \cdot \int_{0}^{x}\left(\rho-2 \sum_{j=1}^{\ell} \alpha\left(t_{j}\right) \cos \left(t_{j} \log u\right)\right) \frac{d u}{\log u},
$$

where $\Pi(x)=\sum \frac{1}{k} \pi\left(x^{1 / k}\right)$. Hence the Chebyshev function

$$
\begin{aligned}
\psi(x) & =\int_{1}^{x} \log u d \Pi(u)=\int_{1}^{x}\left(\rho-2 \sum_{j=1}^{\ell} \alpha\left(t_{j}\right) \cos \left(t_{j} \log u\right)\right) d u+o(x) \\
& =\left(\rho-2 \sum_{j=1}^{\ell} \alpha_{j} \frac{t_{j} \sin \left(t_{j} \log x\right)+\cos \left(t_{j} \log x\right)}{1+t_{j}^{2}}\right) x+o(x),
\end{aligned}
$$

where $\alpha_{j}$ denote $\alpha\left(t_{j}\right)$.

Therefore the condition

$$
\psi(x)=\left(\rho-2 \sum_{j=1}^{\ell} \alpha_{j} \frac{t_{j} \sin \left(t_{j} \log x\right)+\cos \left(t_{j} \log x\right)}{1+t_{j}^{2}}\right) x+O\left(x(\log x)^{-M}\right)
$$

with $M>M_{0}$ should replace the one appearing in the abstract, the condition (1.4) of Theorem 2, and the one in the remark following Corollary 1 of [2].

With the new condition (2), Lemma 3.2 of [2] can be upheld by the following argument and no more change in [2] is needed. Actually, in the proof of the lemma we have

$$
-\frac{\zeta^{\prime}(s)}{\zeta(s)}=\sum_{j=0}^{\infty} \Lambda\left(n_{j}\right) n_{j}^{-s}=\int_{1}^{N} x^{-s} d \psi(x)+\int_{N+}^{\infty} x^{-s} d \psi(x)=I_{1}+I_{2},
$$

say, with $I_{1}$ estimated as before. Then

$$
I_{2}=I_{21}+I_{22}
$$

where

$$
\begin{aligned}
I_{21}= & \int_{N+}^{\infty} x^{-s} d\left(\left(\rho-2 \sum_{j=1}^{\ell} \alpha_{j} \frac{t_{j} \sin \left(t_{j} \log x\right)+\cos \left(t_{j} \log x\right)}{1+t_{j}^{2}}\right) x\right) \\
= & \rho \frac{N^{-s+1}}{s-1}-\sum_{j=1}^{\ell} \alpha_{j}\left(\left(\frac{N^{-s+1+i t_{j}}}{\left(s-1-i t_{j}\right)\left(1+i t_{j}\right)}+\frac{N^{-s+1-i t_{j}}}{\left(s-1+i t_{j}\right)\left(1-i t_{j}\right)}\right)\right. \\
& \left.+i t_{j}\left(\frac{N^{-s+i t_{j}}}{\left(s-i t_{j}\right)\left(1+i t_{j}\right)}-\frac{N^{-s-i t_{j}}}{\left(s+i t_{j}\right)\left(1-i t_{j}\right)}\right)\right)=O(1)
\end{aligned}
$$


for $1 \leq \sigma$ and $t$ with $|t| \geq t_{l}+1$ or $\left|t \pm t_{j}\right|>d, j=0,1, \ldots, \ell$, and

$$
\begin{aligned}
I_{22} & =\int_{N+}^{\infty} x^{-s} d\left(\psi(x)-\left(\rho-2 \sum_{j=1}^{\ell} \alpha_{j} \frac{t_{j} \sin \left(t_{j} \log x\right)+\cos \left(t_{j} \log x\right)}{1+t_{j}^{2}}\right) x\right) \\
& \ll 1+|s| \int_{N}^{\infty} x^{-\sigma-1} O\left(x(\log x)^{-M}\right) d x \ll 1+\frac{|t|(\log N)^{-M+1}}{M-1}
\end{aligned}
$$

for $1 \leq \sigma \leq 2$ by integration by parts. Letting $\log N=|t|^{\frac{1}{M}}$, the result of the lemma follows.

\section{References}

1. Beurling, A.: Analyse de la loi asymptotique de la distribution des nombres premiers généralisés I. Acta Math. 68, 255-291 (1937)

2. Zhang, W.-B.: Mean-value theorems for multiplicative functions on Beurling's generalized integers (I). Math. Z. 251, 359-391 (2005). doi:10.1007/s00209-005-0807-8 